\title{
Linking Diffractive and Geometrical Optics Surface Scattering at a Fundamental Level
}

\author{
Christi Kay Madsen \\ Department of Electrical and Computer Engineering, Texas A\&M University, College Station, TX, USA \\ Email: cmadsen@tamu.edu
}

How to cite this paper: Madsen, C.K. (2022) Linking Diffractive and Geometrical Optics Surface Scattering at a Fundamental Level. Optics and Photonics Journal, 12, 1-17. https://doi.org/10.4236/opj.2022.121001

Received: December 30, 2021

Accepted: January 27, 2022

Published: January 30, 2022

Copyright $\odot 2022$ by author(s) and Scientific Research Publishing Inc. This work is licensed under the Creative Commons Attribution International License (CC BY 4.0).

http://creativecommons.org/licenses/by/4.0/

\begin{abstract}
Optical surface scattering analyses based on diffractive optics (DO) are typically applied to one surface; however, there is a need for simulating surface scattering losses for devices having many surface interactions such as light pipes. Light pipes are often simulated with geometric optics (GO) using ray tracing, where surface scattering is driven by the surface slope distribution. In the DO case, surface scattering analyses depend on the spatial frequency distribution and amplitude as well as wavelength, with the sinusoidal grating as a fundamental basis. A better understanding of the link, or transition, between DO and GO scattering domains would be helpful for efficiently incorporating scattering loss analyses into ray trace simulations. A formula for the rootmean-square (rms) scattered angle width of a sinusoidal reflection grating that depends only on the surface rms slope is derived from the nonparaxial scalar diffraction theory, thereby linking it to GO. The scatter angle's mean and rms width are evaluated over a range of grating amplitudes and periods using scalar theory and full vector simulations from the $\mathrm{COMSOL}^{\circledR}$ wave optic module for a sinusoidal reflection grating. The conditions under which the diffraction-based solution closely approximates the GO solution, as predicted by the rms slope, are identified. Close agreement is shown between the DO and GO solutions for the same surface rms slope scattering loss due to angular filtering near the critical angle of a total internal reflection (TIR) glass-to-air interface.
\end{abstract}

\section{Keywords}

Diffractive Optics, Geometrical Optics, Diffraction Gratings, Surface Scattering, Light Pipes

\section{Introduction}

Optical surface scattering analyses are often conducted using a diffraction-based 
approach for single surface light scattering, as opposed to using geometrical optics approximations (GOA). For applications with numerous surface reflections, such as light pipes based on total internal reflection at the interface, a simpler surface scattering model is desired that can be combined with ray tracing simulations for the overall device. For example, we have fabricated glass light pipes with millimeter-scale cross-sections that are several centimeters in length [1]. The light is guided via total internal reflection as it propagates along the light pipe and undergoes numerous sidewall reflections that vary in number depending on the incident ray angles. We have implemented a geometrical optics (GO) simulation to compare the expected surface scattering loss to our experimental measurement results [2]. Beyond the GO simulation, the next step is to evaluate the potential for diffraction-based scattering effects that could introduce wavelength-dependent losses in the light pipe.

Under the GO approximation, surface scattering is driven by the distribution of the surface slope. In the DO case for smooth surfaces, $\sigma \cos \theta_{0}<0.02 \lambda \quad$ [3] where $\sigma$ is the rms surface roughness and $\theta_{0}$ is the specular reflection angle, the surface scattering depends on the Fourier transform of the PSD. For rough surfaces, the Beckmann-Kirchhoff scatter theory reduces to the geometrical optics approach [3]. For large values of $\sigma$, for example $\sigma \geq 0.3 \lambda$ [4], the DO solutions for the scattered angle distribution are proportional to the rms slope, so the DO and GO solutions are driven by the same surface property. A larger range of applicability for the GO solution was proposed by Tang [5], who defined a criterion for the rms roughness, $\sigma \cos \theta_{0} \geq 0.17 \lambda$, whereby the scattered light distribution from the GO solution compares closely with a full wave solution. Even smaller values for $\sigma / \lambda$ compare well to the wave solution when a different criterion is used. For normal incidence, the standard deviation of the scattered light intensity from a metal surface has been shown to be proportional to the rms slope using a DO analysis [6]. Experimental surface measurement results, including incidence angles up to 40 degrees, confirmed the dependence on slope [7]. More detailed insights are desired to better understand how the surface properties that drive the DO and GO models are related for smooth and moderately rough surfaces to determine when the simpler GOA may be used to estimate surface scattering losses, particularly when many surfaces are encountered.

A goal of this paper is to define a quantitative demarcation line through the two dimensional (2D) sinusoidal reflective grating parameter space to divide between DO and GO relevant scatter analysis domains. Traditionally, the demarcation is based only on grating period relative to wavelength. Little attention is typically given to the dependence on angle of incidence with respect to when diffractive effects are negligible and surface scattering can be adequately approximated with a GOA. An analysis on the rms scatter angular width is provided that quantitatively connects the inputs and outputs of scalar diffraction theory and the GOA. The NP scalar diffraction theory and sinusoidal reflection grating geometry are discussed first, and then a formula for the scatter angular 
width is derived from the NP scalar theory. The necessary approximations and simulation results to evaluate the applicability over a range of amplitudes and grating periods from low- to mid-spatial periods are the focus of the remaining sections.

\section{NP Scalar Solution for a Sinusoidal Reflection Grating}

For optical scattering over a range of smooth to rough surfaces, full vector wave solutions are time consuming, and solutions are needed for a large ensemble of random surfaces to obtain accurate means and standard deviations. To avoid the need for full vector electromagnetic simulations, a nonparaxial (NP) scalar diffraction theory has been developed that underpins a general transfer function approach for modeling diffractive surface scattering, known as the Generalized Harvey-Shack (GHS) model [3]. The GHS model is based on the diffraction from sinusoidal surface variations over a range of spatial frequencies, depending on a Fourier series for deterministic surfaces or power spectral density (PSD) for randomly varying surfaces. While the GHS model overcomes limitations of prior analytic approaches and includes statistical surface representations, it has been implemented predominantly for single surface analysis and light scattering measurement systems.

Harvey and Pfisterer [8] describe a nonparaxial scalar diffraction theory and power conservation conditions for the case of a sinusoidal reflection grating. The grating equation for the $m$ th-order reflection is given as follows using their sign convention:

$$
\beta_{m}+\beta_{i}=\frac{m}{\hat{d}}
$$

where $\theta_{i}$ is the incidence angle, $\theta_{m}$ is the $m$ th-order diffraction angle, $\beta_{i}=\sin \theta_{i}, \quad \beta_{m}=\sin \theta_{m}$, and $m=0, \pm 1, \pm 2, \cdots$. The normalized peak-to-valley grating height and period are given by $\hat{h}=h / \lambda$ and $\hat{d}=d / \lambda$, respectively. For a perfectly reflecting surface, the efficiency for each order is calculated using the mth-order Bessel functions of the first kind:

$$
\eta_{m}=J_{m}^{2}\left(\frac{a}{2}\right)
$$

where the Bessel function argument depends on the order $m$ as follows:

$$
\frac{a}{2}=\pi \hat{h}\left[\cos \left(\theta_{0}\right)+\cos \left(\theta_{m}\right)\right]
$$

For a given set of grating parameters and wavelength, the efficiency $\eta_{m}$ into each order is quickly calculated in MATLAB ${ }^{\circledR}$. An estimate of the number of orders that will contain significant power for a given surface height is helpful in comparing to standard surface quality metrics. The value of $a / 2$ is easily evaluated for a given power in the $m$ th-order, summing the plus and minus order contributions gives $2 \eta_{m}^{2}=2 J_{m}^{2}\left(\frac{a}{2}\right)$. The Bessel function argument corresponding 
to a total power of $1 \%$ in the $|m|>1$ orders under a "locally paraxial" constraint, defined by $\theta_{m} \cong \theta_{0}$ where the diffracted orders are "locally paraxial" with respect to the specular reflection axis, is $a / 2=0.142$. For an incident angle of 45 degrees, $a / 2=\sqrt{2} \pi \hat{h}$. For our numerical examples, the value of $h(\mathrm{~nm})$ for a free space wavelength $\lambda_{0}=500 \mathrm{~nm}$ is calculated assuming incidence in glass with refractive index $n=1.46$. Under these assumptions, a $10 \mathrm{~nm}$ height is relatively smooth and the 0th-order dominates. For order of magnitude $h=100 \mathrm{~nm}$, the 3rd-order is starting to pick up power, but the second and lower orders carry most all the power. At larger incidence angles, and longer wavelengths, larger surface heights will result and vice versa as the incidence angle approaches 0 degrees and for shorter wavelengths.

For a perfectly reflecting surface, the infinite sum over orders for $J_{m}^{2}\left(\frac{a}{2}\right)$ is unity, and power is conserved. For TE polarization, the reflectance for a non-perfectly reflecting surface is calculated as the geometric mean of the Fresnel reflectivity, i.e. $Q_{T E}=\sqrt{R\left(\theta_{0}\right) R\left(\theta_{m}\right)}$, while a different function for $Q_{T M}\left(\theta_{i}, \theta_{m}\right)$ is defined in the literature for the mth-order TM polarization reflectance [9].

\section{Sinusoidal Surface Description}

As seen in the previous section, sinusoids are the basis functions for modeling a random surface using DO, as they provide discrete spatial frequencies and thus diffraction angles. For GO, however, piecewise linear functions are a good basis in $1 \mathrm{D}$ since the surface normal is constant. The simplest function composed of linear pieces that mimics a sinusoid is a triangular function. While the slope is constant over each linear section, a fundamental and several higher order spatial frequencies are associated with the triangular function. A triangular mesh is an example of a useful piecewise planar approximation of a surface in $2 \mathrm{D}$. In practical surface modeling, many spatial frequencies with varying amplitudes are employed to closely approximate a surface. The increase in discrete diffracted angles will lead to a more continuous scatter angular distribution as measured for real surfaces. For simplicity, a single spatial frequency is assumed in the following analysis to compare the DO and GO scattering behavior.

An important metric for estimating sidewall scattering loss using the GOA is the rms surface slope [10]. The rms slope is a surface property, thus independent of optical wavelength, and can be calculated for random and deterministic surfaces. For a sinusoid with period $d$ and peak-to-valley height $h$, the rms slope is given by [11]

$$
m_{s}=\frac{\pi}{\sqrt{2}} \frac{\hat{h}}{\hat{d}}=\frac{\pi}{\sqrt{2}} \frac{h}{d}
$$

The maximum slope is $\sqrt{2}$ times the rms slope. Light pipe sidewall scattering losses increase as the rms slope increases under the GOA [2]. For calibration, relatively smooth surfaces have rms slopes of 0.01 or less [12]. The analyses in 
the next section aim to evaluate when the rms slope is a sufficient metric to capture surface scattering effects.

One period of a sinusoidal grating surface is shown in Figure 1 (a) with $m_{s}=$ $0.05, d=5 \mu \mathrm{m}$, and $h=113 \mathrm{~nm}$. The surface angle with respect to the horizontal,

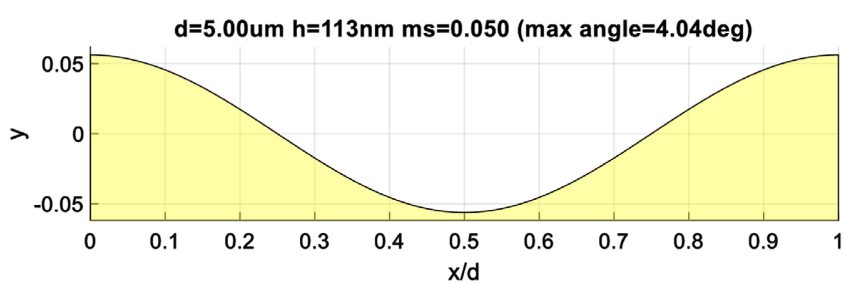

(a)

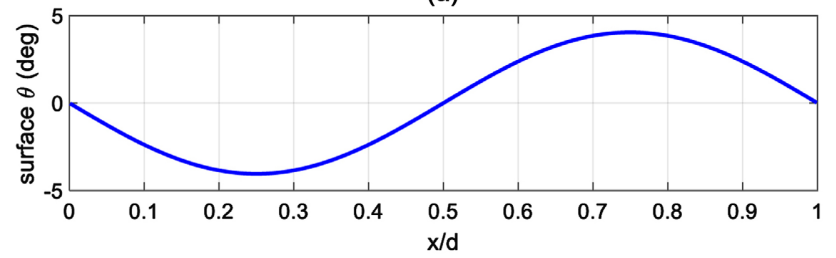

(b)

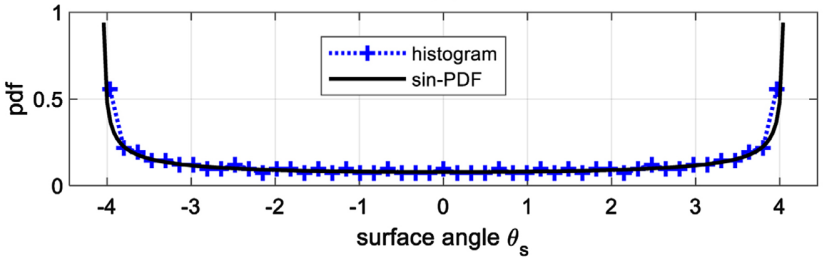

(c)

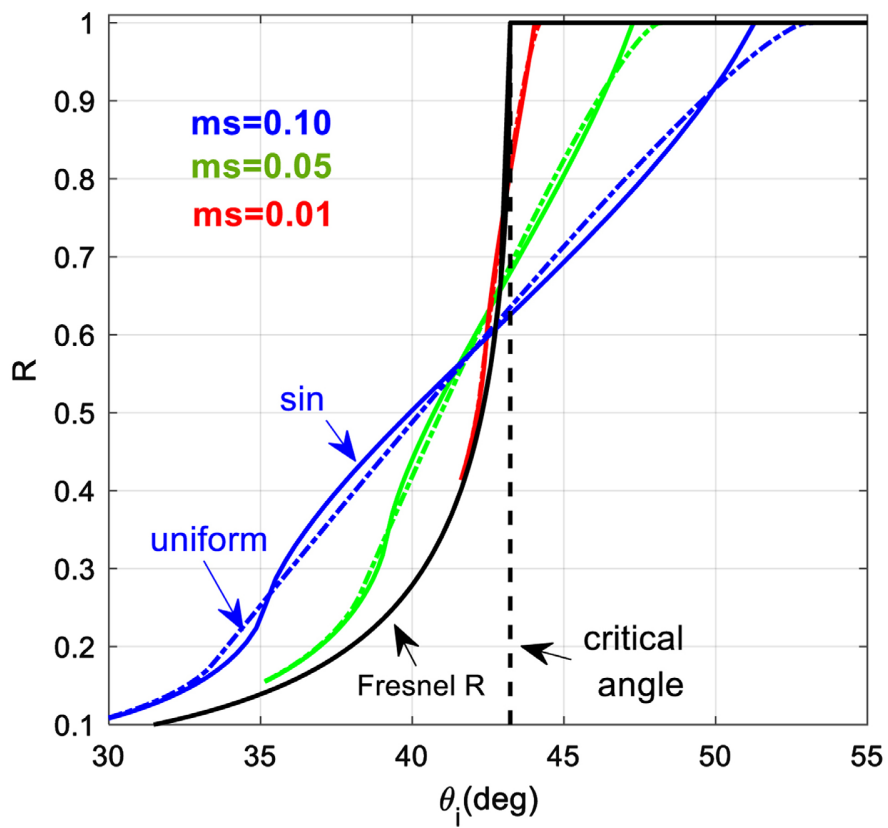

(d)

Figure 1. (a) One period of a sinusoidal grating interface, (b) the local surface angle with respect to the x-axis, (c) surface angle probability distribution function. (d) GOA reflectivity for glass-to-air incidence near the critical angle for both sinusoidal and uniform slope distributions and $m_{s}=0.01,0.05$ and 0.10 . The TE Fresnel specular reflectivity is shown in black. 
shown in Figure 1(b), has a maximum $\max \left(\theta_{s}\right)=\operatorname{atan}\left(\sqrt{2} m_{s}\right)$ which is 4.04 degrees for this example. The distribution of surface angles is shown as a histogram with an overlay of the theoretical probability distribution function in Figure $1(\mathrm{c})$, showing a significant weighting at the extreme surface angles.

Geometrical optics simulations for the reflectivity near the critical angle for a glass-to-air sinusoidal interface are shown in Figure 1(d), highlighting the impact of angular spread on the scattering loss $(R<1)$ versus incidence angle for a TIR interface. The reflectivity is calculated for a sinusoidal slope distribution at three rms slopes, $m_{s}=0.01,0.05$ and 0.10 . The Fresnel reflectivity for the TE polarization, $R_{0}$ (black line), is applied in addition to Snell's law using the slope distributions. We also calculate the results for a uniform distribution of surface slopes (dashed lines) for the same values of rms slope for insight on the impact of the distribution. Since the rms slope for the uniform distribution is $m_{u}=\max (\mathrm{d} y / \mathrm{d} x) / \sqrt{3}$ versus $m_{s}=\max (\mathrm{d} y / \mathrm{d} x) / \sqrt{2}$ for the sinusoidal distribution, equating the rms slopes $\left(m_{u}=m_{s}\right)$ causes the maximum slopes to be different. The variation caused by different slope distributions is most evident in the reflectivity for the larger rms slopes; however, the deviations caused by the different distributions appear to cancel if averaged over the incidence angle range.

\section{A Parametric Bridge from Diffractive to Geometrical Optics}

From the NP scalar theory for a sinusoidal reflection grating, it is not evident how a diffractive optics solution that is wavelength dependent may converge to a wavelength-independent GOA solution, depending only on the surface slope distribution and not on the specific amplitude and period. Neglecting the small impact of material dispersion, the GOA solution assumes the angular spread is identical for all wavelengths, as governed by Snell's law. Intuitively, as the period (d) increases, the diffraction orders are more closely spaced and the grating height $(h)$ can be quite large while having a relatively small $\mathrm{rms}$ angular width. This describes the rough surface limit. The smooth surface dependence on surface slope is not evident.

We begin by exploring the diffracted angular range on reflection, to determine if or when it is independent of wavelength. First, we define an rms width $\delta_{M}$ over the diffraction order $\mathrm{m}$ values, weighted by the order efficiencies as follows:

$$
\delta_{M}^{2}=\frac{2 \sum_{m=1}^{M} m^{2} \eta_{m}}{\eta_{0}+2 \sum_{m=1}^{M} \eta_{m}}
$$

where $M$ is the highest propagating order. When $\delta_{M}$ is calculated and plotted over a range of $a / 2$ values, the result is linear in $\mathrm{a} / 2$ as follows:

$$
\delta_{M}=\frac{1}{\sqrt{2}} \frac{a}{2} \cong \frac{1}{\sqrt{2}} \pi \hat{h}\left[2 \cos \left(\theta_{0}\right)\right]
$$

As noted in Equation (3), a depends on the order in general. The last equality 
employs a "locally paraxial" constraint where the definition of a becomes independent of order $\mathrm{m}$. Going beyond the numerical simulation, a proof is outlined using the following Bessel function recursion equation and sum of product relationships [13]:

$$
\begin{gathered}
\frac{2 m}{x} J_{m}(x)=J_{m-1}(x)+J_{m+1}(x) \\
\sum_{m=-\infty}^{m=+\infty} J_{m}^{2}(x)=1 \\
\sum_{m=-\infty}^{+\infty} J_{m}(x) J_{m+n}(x)=0 \text { for } n= \pm 1, \pm 2, \cdots
\end{gathered}
$$

Squaring the recursion relation and summing over the orders provides the rms width relationship:

$$
\sum_{m=-\infty}^{+\infty} \frac{4 m^{2}}{x^{2}} J_{m}^{2}(x)=\sum_{m=-\infty}^{+\infty}\left[J_{m-1}^{2}(x)+J_{m+1}^{2}(x)+2 J_{m-1}(x) J_{m+1}\right]
$$

Taking $4 / x^{2}$ outside of the sum on the left, with the right-side summing to 2 , and rearranging terms yields

$$
\sum_{m=-\infty}^{+\infty} m^{2} J_{m}^{2}(x)=\frac{x^{2}}{2}
$$

Under the "locally paraxial" approximation, $\hat{d}$ is large and $\gamma_{m} \cong \gamma_{0}=\cos \theta_{0}$. For a constant value of $x=a / 2$, i.e. independent of $m$, the rms order width is

$$
\sigma_{m}=\sqrt{\sum_{m=-\infty}^{+\infty} m^{2} J_{m}^{2}\left(\frac{a}{2}\right)}=\frac{a}{2 \sqrt{2}}
$$

As $\theta_{0} \rightarrow 90^{\circ}$, the sum over the negative $m$-values is truncated due to nonpropagating orders and the above summation limits would need to be modified accordingly. We proceed for angles under which the propagating orders are symmetric and the above equation is satisfied. The angular width can be determined from $\sigma_{m}$. First, the width in directional cosine space, $\sigma_{\beta}$, is related to the diffracted order width by

$$
\sigma_{\beta}=\frac{\sigma_{m}}{\hat{d}} \cong 2 \gamma_{0} \frac{\pi}{\sqrt{2}}\left(\frac{h}{d}\right)=2 \gamma_{0} m_{s}
$$

Then, the diffraction pattern angular half-width, $\sigma_{\theta}$, can be defined from the grating equation using $\sigma_{\beta}$ as follows:

$$
\begin{gathered}
\sin \left(\theta_{0}\right)+\sigma_{\beta}=\sin \left(\theta_{0}+\sigma_{\theta}\right) \\
\sin \left(\theta_{0}+\sigma_{\theta}\right) \cong \sin \theta_{0}+\cos \left(\theta_{0}\right) \sigma_{\theta}
\end{gathered}
$$

The Taylor series expansion for $\sin \left(\theta_{0}+\sigma_{\theta}\right)$ to first order around $\sin \left(\theta_{0}\right)$ is given in Equation (13). Comparing to Equation (12), we find

$$
\sigma_{\beta} \cong \gamma_{0} \sigma_{\theta}
$$

As $\sigma_{\theta}$ increases, higher order terms would need to be retained. Note that $\sigma_{\beta}$ and $\sigma_{\theta}$ are independent of wavelength. The diffracted angle half-width 
equals twice the rms slope $m_{s}$ for a sinusoidal surface corrugation,

$$
\sigma_{\theta} \cong 2 m_{s}
$$

compared to $\sigma_{\theta}=\operatorname{atan}\left(2 m_{s}\right) \cong 2 m_{s}$ in the GOA. For $m_{s}=0.10$, the next higher order term in the Taylor series expansion is proportional to $\frac{\sigma_{\theta}^{2}}{2} \cong 0.02$ so the approximation is reasonable for rms slopes around 0.1 or less.

\section{NP-DO Simulations: Deviations in Angular Mean and Width}

Using the Bessel function efficiencies and $a / 2$ dependence on both the specular and diffracted angles, we calculated the rms order width $\delta_{M}$ and compare it to Equation (10) for $\sigma_{m}$ (dashed black line) in Figure 2. Graphs are shown for constant $\hat{h}=0.175$ and various slopes. The $\cos \theta_{0}$ dependence is evident until the cutoff of the $m=-1$ order, leaving only the specular and +1 order and shifting the mean. The average order $m_{\text {avg }}$, ideally zero, is shown in dotted lines to increase up to the first-order cutoff and then decrease for each slope. Using the sign convention from [8], the incidence angle cutoff for the $m=-1$ order, $\theta_{c 01}$, is given by

$$
\sin \theta_{c o 1}=1-\frac{1}{\hat{d}}
$$

For angles larger than this cutoff, only the +1 (and higher orders if $\hat{h}$ is

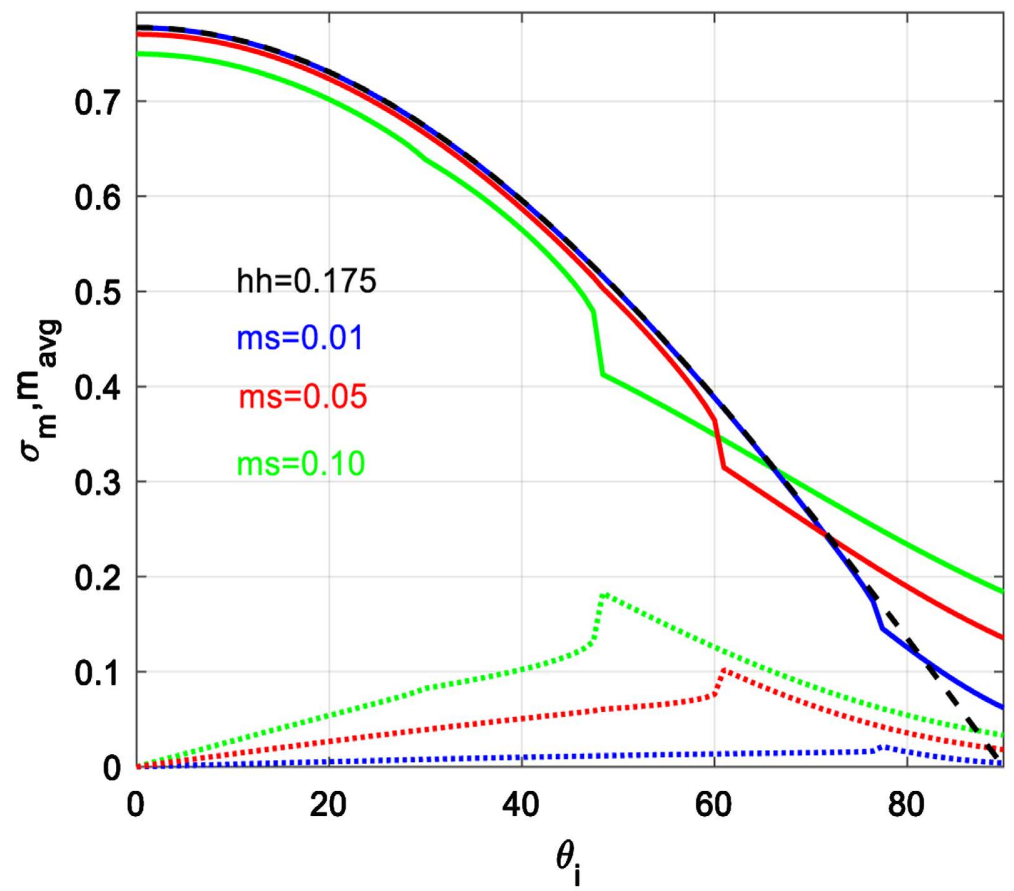

Figure 2. The diffraction order rms width $\sigma_{m}$ (solid lines) and average $m_{\text {avg }}$ (dotted lines) versus input angle (degrees) for a glass-metal interface for slopes of 0.1 (green), 0.05 (red) and 0.01 (blue). The theoretical prediction is shown in the black dashed line for the rms width. 
larger) and 0th orders are diffracted. When only one side of the diffraction orders is propagating, as expected, the results deviate from our above analysis.

The angular width is calculated from Equations (11) and (14) as follows and plotted in Figure 3(a) for multiple slopes:

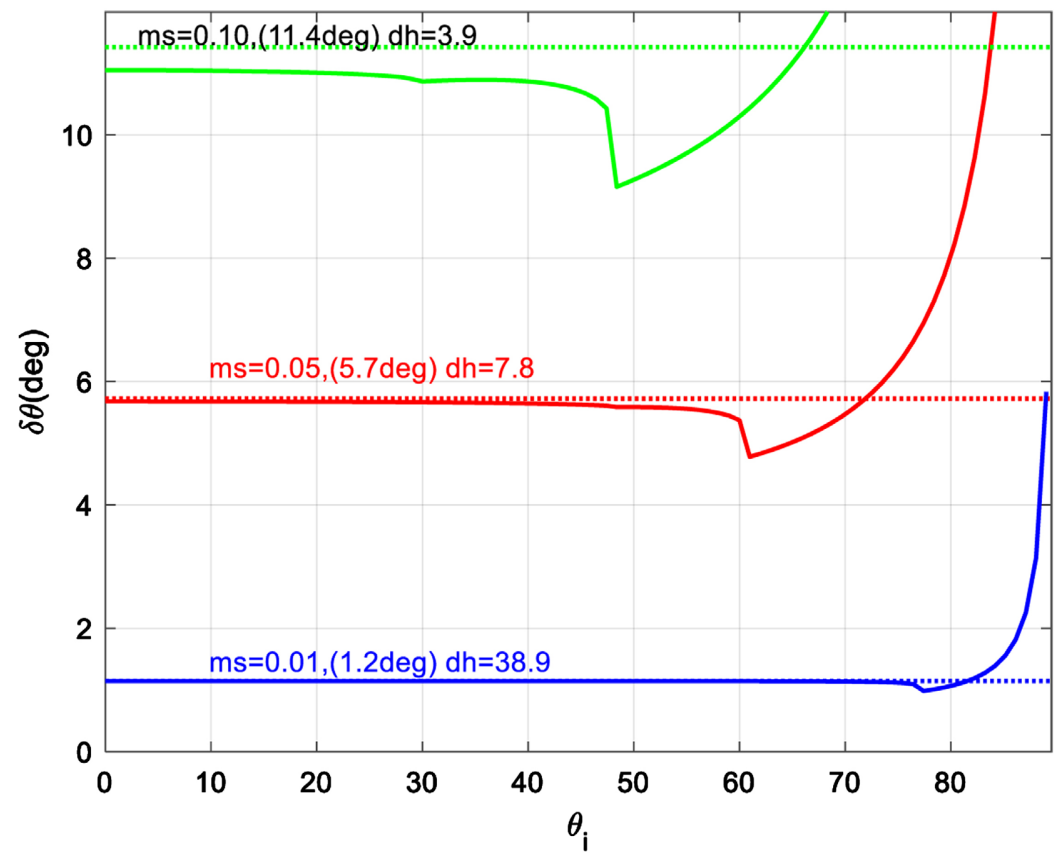

(a)

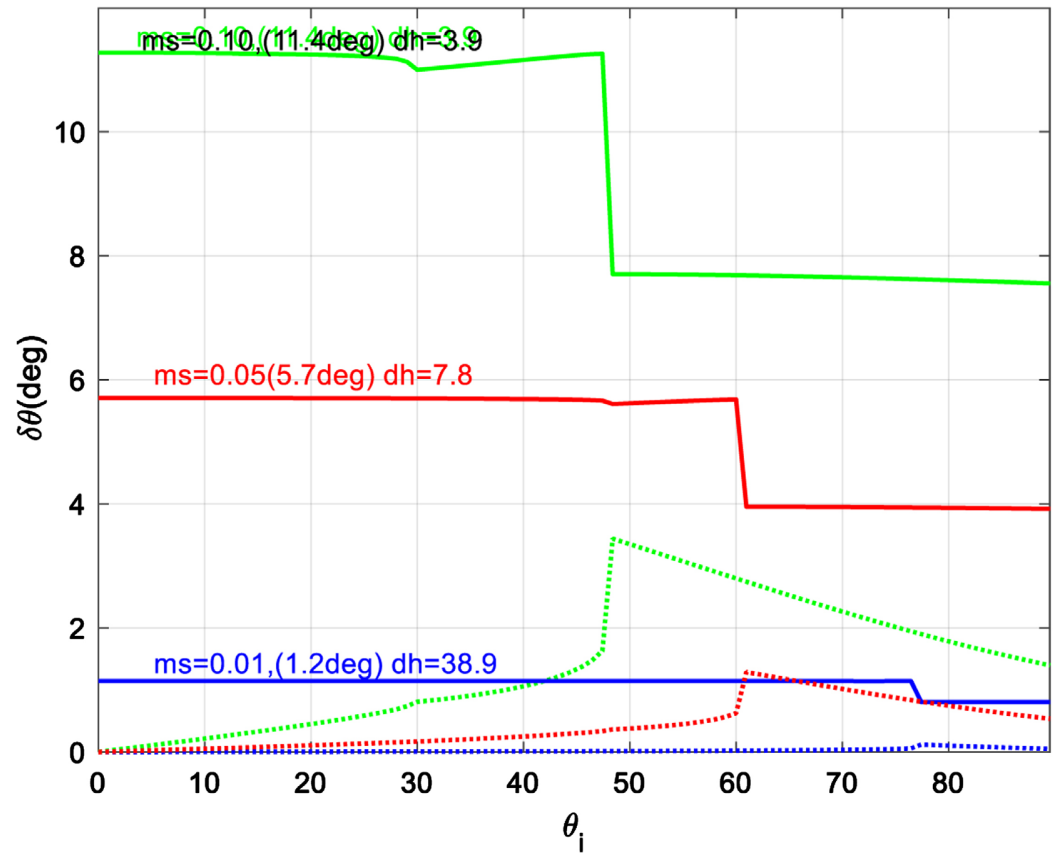

(b)

Figure 3. Angular rms width (degrees) over a range of incidence angles (degrees) and slopes $(0.01,0.05$ and 0.1$)$ for $\hat{h}=0.175$ : a) calculation from rms order width and b) direct calculation of angular rms width (solid) and mean angle deviation (dotted). 


$$
\delta \theta=\frac{\delta_{M}}{\hat{d} \cos \theta_{i}}
$$

While the general behavior is consistent with $\sigma_{\theta} \cong 2 m_{s}$ shown in dashed lines, there are significant deviations as the incidence angle approaches the $m=-1$ cutoff and beyond. The $1 / \cos \theta_{i}$ dependence is problematic in this region. To avoid this singularity, the angular rms width and mean were calculated directly using the scalar theory diffracted order efficiencies and plotted in Figure 3(b). A constant angular width results over a range of incidence angles using the NP scalar theory.

Calculations for other $\hat{h}$ values show the same behavior except the $m=-1$ cutoff is shifted due to a different $\hat{d}$ value for a given slope, as shown in Figure 4(a). The angular width is predicted by the surface rms slope, indicating that the geometrical optics behavior is achieved. As $\hat{d}$ increases, the range of incidence angles over which the GOA is valid increases. We have simulated cases where only a few orders propagate, which highlight the $m=-1$ cutoff condition as a transition region between the GOA and diffractive optics behavior. The resulting scatter distribution becomes asymmetric which is more dramatic for a small number of propagating orders, i.e. small $\hat{h}$. Decreases in $\hat{d}$ move the $m=-1$ cutoff further away from 90 degrees, toward normal incidence.

To quantify the shift in the mean scatter angle $\theta_{\text {avg }}$ from the specular angle, let $\Delta \theta_{\text {avg }}=\theta_{0}-\theta_{\text {avg }}$, assuming positive values for $\theta_{0}$. Figure 4 (b) shows the calculated relative deviation of the mean scatter angle $\Delta \theta_{\text {avg }} / \sigma_{\theta}$ over a range of slopes, for $\hat{h}=0.7$ and $\hat{h}=0.175$ at each slope.

Limiting the incidence angle, which scales with the rms slope, will effectively limit the mean scatter angle deviation across a range of $\hat{h}$ and $\hat{d}$ values. For further calculations, let the $5 \%$ mean angle deviations normalized to the rms width, $\Delta \theta_{\text {avg }} / \sigma_{\theta} \leq 0.05$, limit the acceptable mean angle deviation. Then, the resulting incidence angle limit is given by $\theta_{i, \max }$. For $\theta_{i} \leq \theta_{i, \max }$, the rms width approximates the GOA without a significant change in the mean scatter angle.

A plot of the maximum incidence angle $\theta_{i, \max }$ versus period $\hat{d}$ is shown in Figure 5 corresponding to the range of angles under which the GOA is valid. Three values of $\hat{h}$ are simulated using the nonparaxial scalar theory, $\hat{h}=0.175$, 0.3 and 0.45. Four slopes, $m_{s}=0.01,0.03,0.05$, and 0.10 , are simulated for each $\hat{h}$. Each marker indicates a calculated point using the NP scalar theory.

The GOA is examined using two different criteria on deviations from the NP scalar theory. For the first criterion, the solid curves show the maximum input angle for a 5\% deviation in the mean scatter angle relative to the rms scatter width, which is the stricter criterion. In this case, the slope drives the maximum incidence angle, with very little dependence on the $\hat{h}$ value.

For the second criterion, the markers without lines represent the maximum input angle for a 5\% deviation in the scatter angle width from the rms slope estimate. For comparison, the dashed and dotted lines represent the cutoff of the 


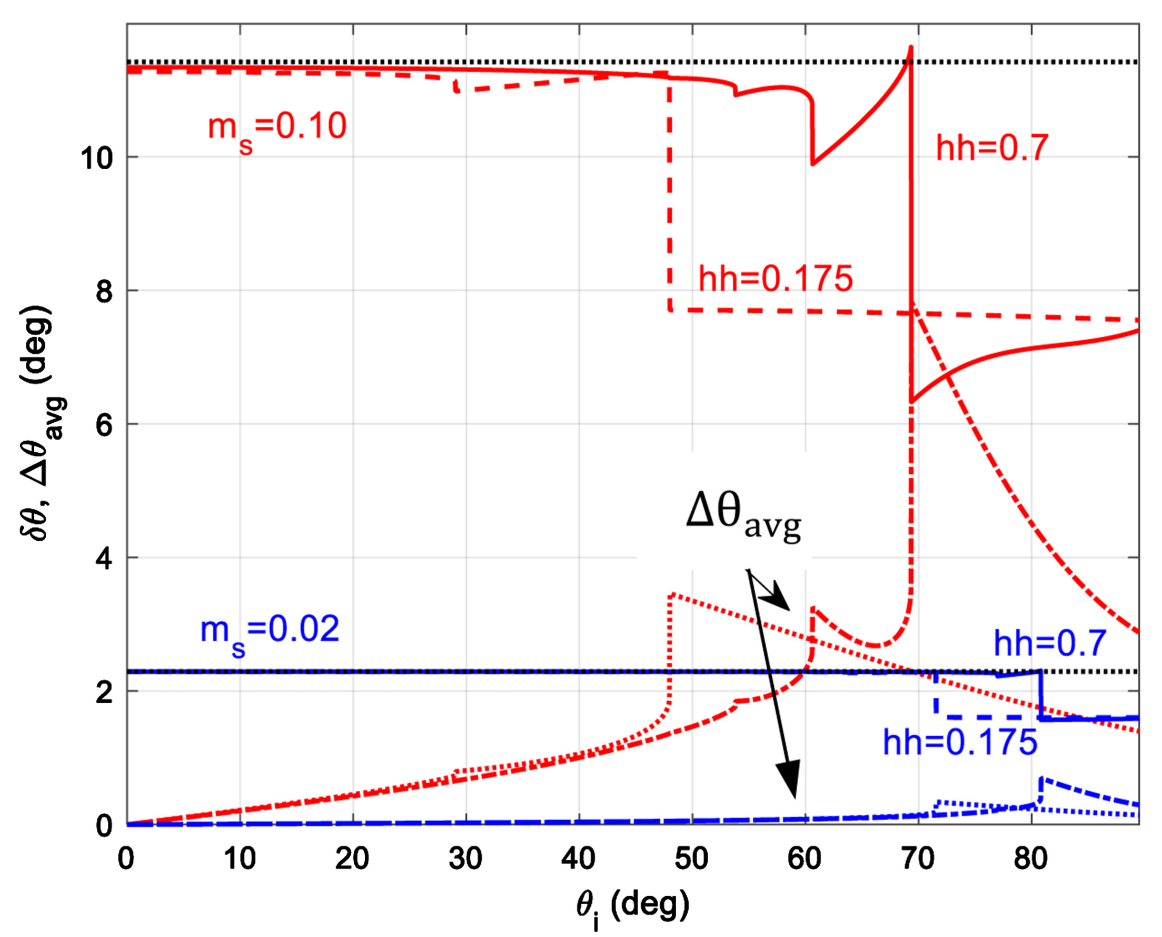

(a)

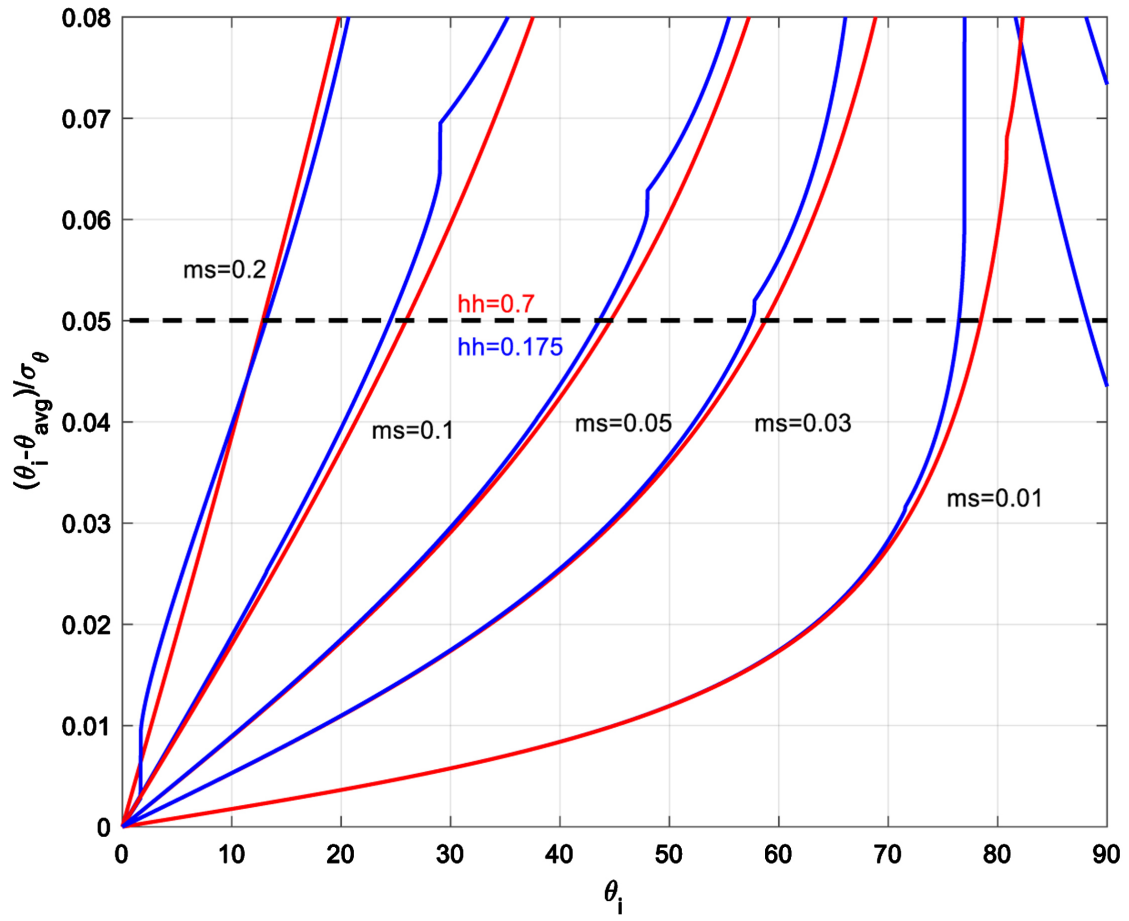

(b)

Figure 4. (a) Angular rms width using scalar diffraction theory versus incidence angle for $m_{s}=0.10$ (red) and 0.02 (blue), with $\hat{h}=0.7$ (solid) and $\hat{h}=0.175$ (dashed). The deviation of the mean scatter angle $\Delta \theta_{\text {avg }}$ is shown in the dotted $(\hat{h}=0.175)$ and dash-dot ( $\hat{h}=0.7$ ) curves. (b) Deviation of mean scatter angle $\Delta \theta_{\text {avg }}$ over a range of $m_{s}$ values from 0.01 to 0.2 , with $\hat{h}=0.7$ (red) and $\hat{h}=0.175$ (blue). All angles are in degrees and $h h=\hat{h}$. 


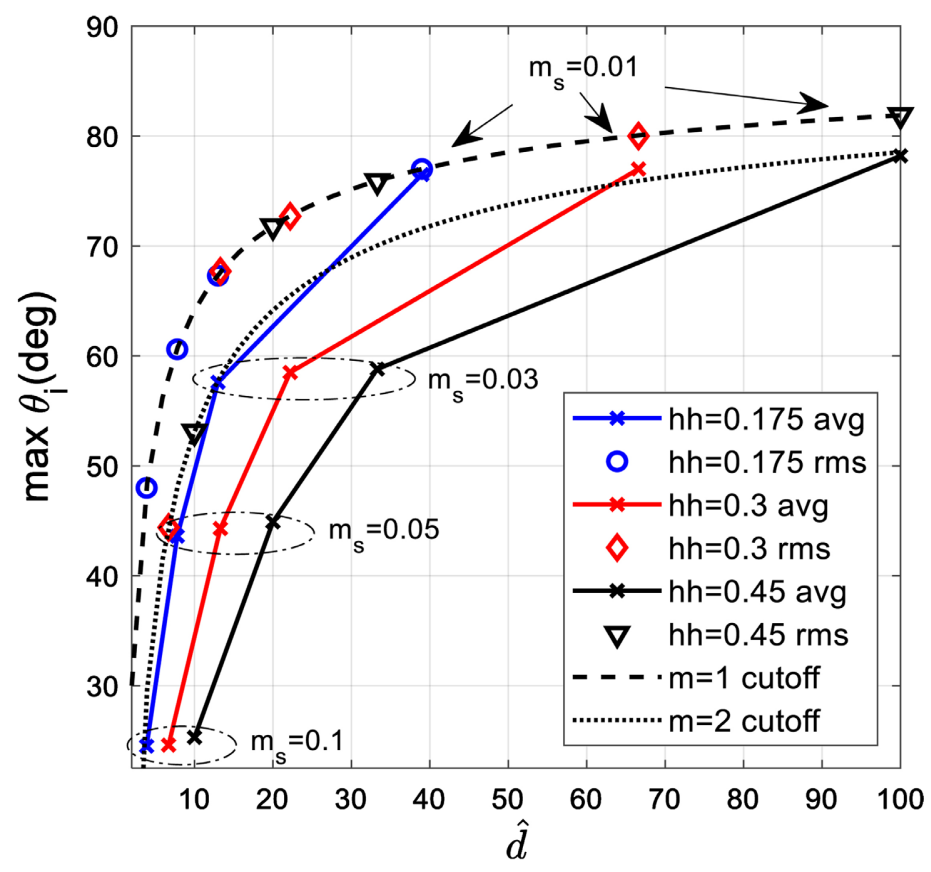

Figure 5. The solid curves show the maximum input angle for a 5\% deviation in the mean scatter angle relative to the rms scatter width. The markers, without lines, represent the maximum input angle for a $5 \%$ deviation in the scatter angle width from the rms slope estimate. The dashed and dotted lines represent the cutoff of the $1^{\text {st }}$ and $2^{\text {nd }}$ diffraction orders. The corresponding slope $m_{s}$ is shown for the solid curve points.

first and second diffraction orders, i.e. $\theta_{i, \max }=\operatorname{asin}\left(1-m / \hat{d}_{\min }\right)$ for $m=1$ and 2 . For this deviation in spectral width criterion, the incidence angle for the first-order cutoff is the major driver for the GOA to be applicable, as most of the simulated points fall on the dashed line. For the largest slope case, $m_{s}=0.1$, and $\hat{h}>0.175$, the simulated points fall on the second-order cutoff as expected from the simulations in Figure 4(a).

For small slopes, indicating smooth surfaces, the first diffraction order cutoff serves as a good limit for setting the input angle range to satisfy the GOA. Both criteria converge to this result; however, smooth surface scattering is typically discussed in the DO domain without comment on the relevance for a GOA solution. Further discussion is given in the next section.

For a large range of periods occupying the mid-spatial frequency range, the spectral width predicted by the GOA applies over a relatively large range of incidence angles that can be estimated from the first diffraction-order cutoff. The applicability as the surface roughness increases is not well-predicted by the NP scalar theory as it becomes less of a good predictor for the diffraction order efficiencies as $\hat{h}$ increases. Thus, we confirm the GOA domain using COMSOL in Section 7.

\section{Discussion on the Smooth Surface Limit}

In the limit of small slopes, implying geometric smoothness, we compare the 
above "rms" analysis to the smooth surface regime of diffractive optics solutions. In the smooth surface approximation, both the Rayleigh-Rice and the Generalized Harvey-Shack scatter theory show the angular scattered intensity is proportional to the power spectral density [9] of the surface height deviation around the surface mean height.

For a sinusoidal surface, the scattered intensity occurs at the predicted $+/-$ 1 st-order angles with a single-sided angular spread of $\lambda / d$ under the near-normal incidence angle approximation. The magnitude for each $m=1$ order is $(a / 4)^{2}$ and thereby proportional to $(h / \lambda)^{2}$; however, the limit on the amplitude for this approximation is very restrictive, $h \leq 0.07 \lambda$ for $5 \%$ error or less [9]. Based on this information, it doesn't appear that the GOA would apply.

If the smooth surface limit is observed in terms of the rms width $\sigma_{\theta}$ under "locally paraxial" conditions, we have shown that it depends only on $m_{s}$ and is wavelength independent over a wide range of conditions, including a much larger range of amplitudes than $h \leq 0.07 \lambda$ (see Figure 5(b)). The rms slope provides the basic input surface parameter and rms angular width links the results between the DO and GO analysis. Thus, some smooth surfaces can be modeled effectively using a GOA while others require a DO analysis, under conditions noted in the previous section to be driven by the first diffraction order cutoff.

\section{Wave Optic Simulations and Reflection at a TIR Interface}

To support the NP scalar diffraction calculation results, we used COMSOL wave optics $2 \mathrm{D}$ to simulate the TE response for a few grating parameters using a glass-silver interface for the reflecting surface. One grating period was simulated using periodic boundary conditions and ports that calculated the diffraction order powers. Vector simulations of diffraction order efficiency over a full range of incidence angles were used to calculate an rms scatter angle width and mean scatter angle deviation from the specular angle. The results are shown for $\hat{d}=7.77$ with $\hat{h}=0.175$ and $m_{s}=0.05$ in Figure $6(\mathrm{a})$ and with $\hat{h}=0.35$ and $m_{s}=0.10$ in Figure $6(\mathrm{~b})$. The key features predicted from the NP scalar diffraction theory are confirmed, including the $m=-1$ cutoff impact on the rms width and the deviation of the mean scatter angle. The incidence angles for which the mean deviation $\Delta \theta_{\text {avg }}$ and rms width $\sigma_{\theta, N P}$ vary by $5 \%$ of the GOA width $\sigma_{\theta, g}$ are noted. The vector simulation results deviate from the NP scalar diffraction behavior as the incidence angle approaches 90 degrees. Modifications for the NP scalar theory to address this discrepancy are covered in a separate paper [14]. With the modifications, the NP scalar theory was shown to closely estimate the wave simulation diffracted power in each order over the full range of incidence angles for relative periods $\hat{d} \geq 2$, heights $\hat{h} \leq 0.5$, and slope $m_{s} \leq 0.20$.

Using the $\mathrm{COMSOL}^{\circledR}$ wave optics module in $2 \mathrm{D}$, we simulated TE diffraction in reflection at a glass-air interface with a sinusoidal surface corrugation, assuming 


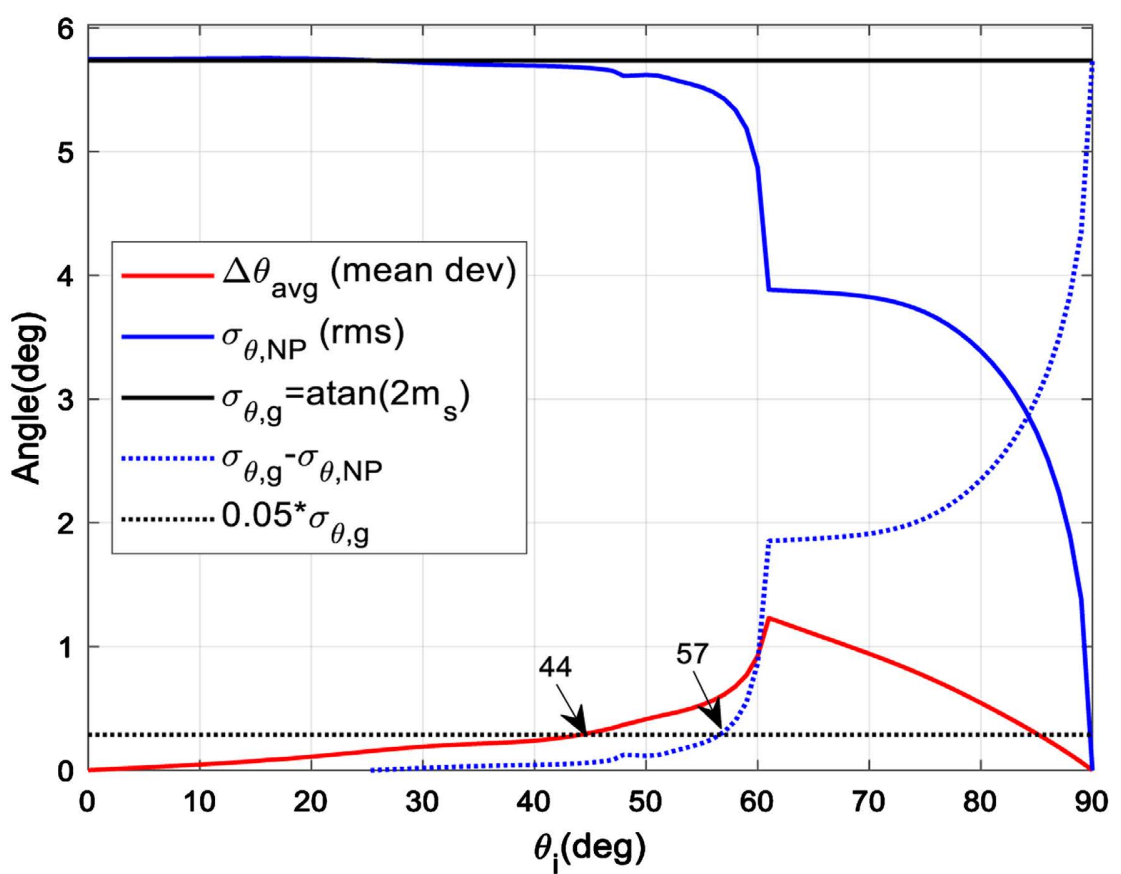

(a)

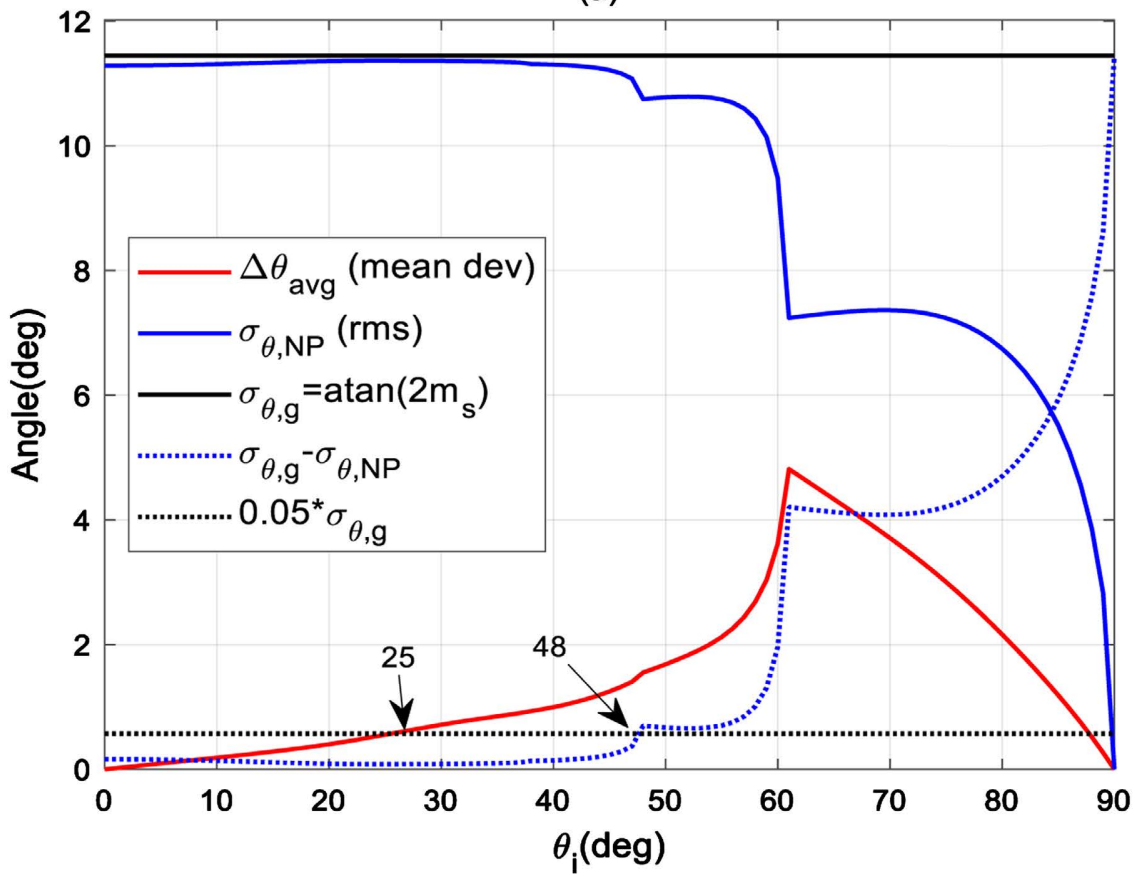

(b)

Figure 6. Calculation of angular rms width $\left(\sigma_{\theta}\right)$ and deviation of the scatter angle mean $\left(\Delta \theta_{\text {avg }}\right.$ ) from the specular angle based on $\mathrm{COMSOL}^{\oplus}$ wave simulation for (a) $\hat{h}=0.175$ and $m_{s}=0.05$ and (b) $\hat{h}=0.35$ and $m_{s}=0.10$. The deviation criterion is set at $5 \%$ of the GOA angular rms width $\sigma_{\theta, g}$. Both have $\hat{d}=7.77$.

$n=1.46$ for the glass refractive index. The simulation results are shown in Figure 7 for two different slopes, $m_{s}=0.06$ and $m_{s}=0.024$, and relatively large heights of $\hat{h}=0.642$ and 0.584 , respectively. The estimated loss around the 


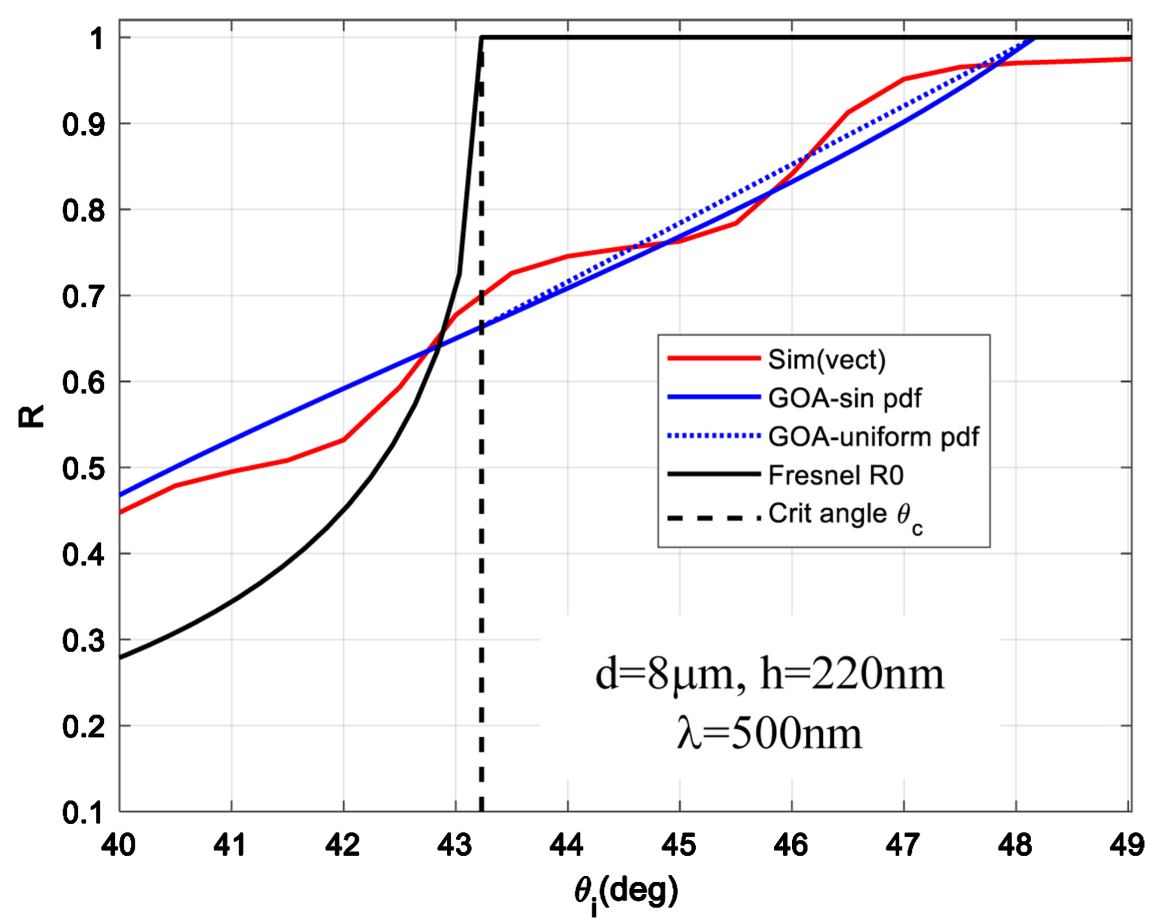

(a)

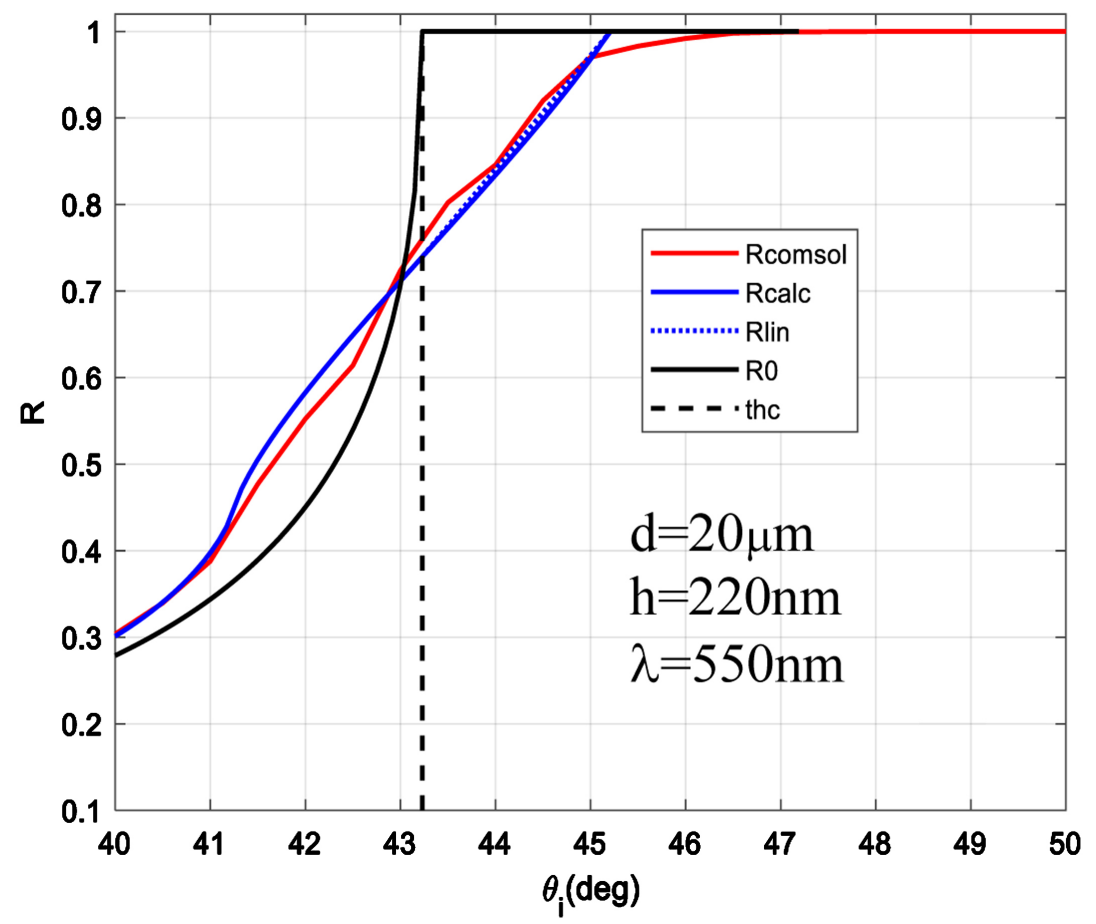

(b)

Figure 7. Vector wave simulations $\left(\mathrm{COMSOL}^{\oplus}\right)$ compared to GOA for a glass $(n=1.46)$ to air interface with (a) $\hat{h}=0.642, \hat{d}=23.4$ and $m_{s}=0.06$ and (b) $\hat{h}=0.584$, $\hat{d}=53.1$ and $m_{s}=0.024$. The critical angle (thc) is shown in the dashed line.

critical angle is predicted using a GOA for the sinusoidal surface. The Fresnel reflectivity around the specular angle was incorporated in the GOA simulations. 
The critical angle is shown as well as the Fresnel reflectivity $R_{0}$ for $m=0$. The oscillatory behavior of the DO solution around the GO solution is to be expected since its scatter angle distribution is discrete rather than continuous. Their overlap, or agreement in an average sense with respect to incidence angle, indicates that the rms width is a reasonable metric for comparing the scattering loss influence from different surface distributions. The agreement in DO and GO solutions will be closer when many spatial frequencies are used to approximate a surface instead of this basic comparison using a single spatial frequency function.

\section{Conclusions}

Based on the non-paraxial scalar theory, both $\hat{d}$ and $\hat{h}$ are needed to evaluate the diffraction efficiencies and order angular dependence. So, one geometry ( $\mathrm{d}$ and $\mathrm{h}$ ) yields different scatter angle distributions at different wavelengths. In this paper, we derived a formula for the rms scattered angular width from the basic diffraction formula, showing how it resulted from the application of a "locally paraxial" approximation that directly yields the geometrical optics solution, i.e. the diffractive optics solution becomes independent of wavelength and the scattered angle width is predicted by the surface slope rms width.

Both the scattered angular width and deviation of the mean scatter angle were shown to be important metrics in comparing DO and GO solutions, as they would impact scattering loss estimates for TIR interfaces, such as for light pipes. Limits were derived on the incidence angle range as a function of the relative grating period, demarcating the GO range of applicability. The requirements for GO to apply for smooth surface domains depend on the normalized period, predominantly, as it affects the first diffraction order cutoff. The shift in the mean scatter angle is also important, particularly for moderately rough surfaces, as it was shown to depend on the rms slope. The ability to predict the rms scatter width over a range of grating parameters, including small amplitudes, provides a direct connection between the GOA and diffraction results. For grating parameters where the GOA condition is satisfied, ray tracing simulations are expected to be sufficient for scattering loss analyses.

\section{Conflicts of Interest}

The author declares no conflicts of interest regarding the publication of this paper.

\section{References}

[1] Madsen, C.K., Dogan, Y., Morrison, M., Hu, C. and Atkins, R. (2018) Glass Light Pipes for Solar Concentration. Laser-Based Micro- and Nanoprocessing XII, 10520, Article ID: 105201A. https://doi.org/10.1117/12.2291249

[2] Madsen, C.K. and Lin, J. (2020) Light Pipes for CPV: Simulation and Measurement of Sidewall Loss Mechanisms. AIP Conference Proceedings, 2298, Article ID: 020006. https://doi.org/10.1063/5.0032994 
[3] Schröder, S., Duparré, A., Coriand, L., Tünnermann, A., Penalver, D.H. and Harvey, J.E. (2011) Modeling of Light Scattering in Different Regimes of Surface Roughness. Optics Express, OE, 19, 9820-9835. https://doi.org/10.1364/OE.19.009820

[4] Vorburger, T.V., Marx, E. and Lettieri, T.R. (1993) Regimes of Surface Roughness Measurable with Light Scattering. Applied Optics, AO, 32, 3401-3408. https://doi.org/10.1364/AO.32.003401

[5] Tang, K., Dimenna, R.A. and Buckius, R.O. (1996) Regions of Validity of the Geometric Optics Approximation for Angular Scattering from Very Rough Surfaces. International Journal of Heat and Mass Transfer, 40, 49-59. https://doi.org/10.1016/S0017-9310(96)00073-7

[6] Rakels, J.H. (1989) Recognised Surface Finish Parameters Obtained from Diffraction Patterns of Rough Surfaces. Surface Measurement and Characterization, 1009, 119-125. https://doi.org/10.1117/12.949162

[7] Cao, L., Vorburger, T.V., Lieberman, A.G. and Lettieri, T.R. (1991) Light-Scattering Measurement of the Rms Slopes of Rough Surfaces. Applied Optics, AO, 30, 32213227. https://doi.org/10.1364/AO.30.003221

[8] Harvey, J.E. and Pfisterer, R.N. (2019) Understanding Diffraction Grating Behavior: Including Conical Diffraction and Rayleigh Anomalies from Transmission Gratings. $O E$, 58, Article ID: 087105. https://doi.org/10.1117/1.OE.58.8.087105

[9] Harvey, J.E. (2019) Understanding Surface Scatter: A Linear Systems Formulation. SPIE. https://doi.org/10.1117/3.2530114

[10] Madsen, C.K. and Hu, C. (2020) Empirical Model for Estimating Light Pipe Sidewall Scattering Loss.

https://www.osapublishing.org/abstract.cfm?uri=FiO-2020-JW6A.12

[11] Stover, J.C. (2012) Optical Scattering: Measurement and Analysis. Third Edition, Stover Publications, Bellingham, Wash. https://spie.org/Publications/Book/975276?SSO=1 https://doi.org/10.1117/3.975276

[12] Hu, C., Dogan, Y. and Madsen, C. (2017) A Better Predictor for Waveguide Outcoupling by Surface Texturing or Roughness. Presented at the Optics for Solar Energy (SOLAR), Boulder, CO. https://doi.org/10.1364/OSE.2017.RW4A.5

[13] Martin, P.A. (2007) On Functions Defined by Sums of Products of Bessel Functions. Journal of Physics A: Mathematical and Theoretical, 41, Article ID: 015207. https://doi.org/10.1088/1751-8113/41/1/015207

[14] Madsen, C.K. (2021) Nonparaxial Scalar Diffraction Theory Modifications for Improved Efficiency Estimation. Applied Optics, AO, 60, 2695-2703. https://doi.org/10.1364/AO.417696 